\title{
An MHD model of Ganymede's mini-magnetosphere suggests that the heliosphere forms in a sub-Alfvénic flow
}

\author{
Margaret Galland Kivelson ${ }^{1,2}$ and Xianzhe $\mathrm{Jia}^{2}$ \\ Received 16 June 2013; revised 19 October 2013; accepted 22 October 2013; published 12 November 2013.
}

[1] Directional fluxes of energetic neutral atoms (ENAs) measured by the Interstellar Boundary Explorer spacecraft reveal a Ribbon of strong emission whose source lies beyond the termination shock. Intense emissions of ENAs (energies 0.1 to $6 \mathrm{keV}$ ) appear along an extended arc significantly displaced from the nose of the heliosphere, the point on the boundary defined by the Sun's motion relative to the local interstellar medium (LISM). The locus of the Ribbon differs from expectations based on early models of the interaction of the solar wind with the LISM, assumed to flow at a super-Alfvénic speed. Here we argue that the distribution of the ENA source can be understood if the flow is sub-Alfvénic. We use a magnetohydrodynamic model of the mini-magnetosphere of Ganymede, embedded in the sub-Alfvénic flow of Jupiter's magnetospheric plasma, to establish where heated ions are distributed on the magnetopause. If the flow of the LISM is sub-Alfvénic, reconnection would occur along an arc centered away from the nose for an appropriately chosen field orientation. Charge exchange with ions heated by reconnection would produce an ENA source distributed in a manner close to that observed. Heating of ions by reconnection can account also for the way ENA images vary with energy. Sub-Alfvénic flow implies not only that reconnection on the heliopause can be centered well away from the nose, but also that no bow shock forms upstream of the heliopause. It also seems probable that the configuration of the heliosphere differs from the bullet shape frequently illustrated.

Citation: Kivelson, M. G., and X. Jia (2013), An MHD model of Ganymede's mini-magnetosphere suggests that the heliosphere forms in a sub-Alfvénic flow, J. Geophys. Res. Space Physics, 118, 6839-6846, doi:10.1002/2013JA019130.

\section{Introduction}

[2] The spatial scales of solar system magnetospheres vary from the thousands of kilometers that characterize Ganymede's magnetosphere to the hundreds of astronomical units (AU) that characterize the heliosphere, the cavity confining the solar wind within the magnetized plasma of the local interstellar medium (LISM). The heliopause, the boundary between the solar wind and the LISM, lies closest to the Sun at the nose, defined by the direction of the Sun's motion (at $\sim 23 \mathrm{~km} \mathrm{~s}^{-1}$ [McComas et al., 2012a]) relative to the LISM. Considerable insight into the properties of the heliosphere has been provided by data from the Voyager 1 and 2 spacecraft, which are moving away from the Sun along trajectories that have reached $\sim 100$ AU somewhat north (V1) and south (V2) of the nose direction. In their outward progress, these spacecraft have crossed an internal shock, the termination shock, beyond which the solar wind flow becomes submagnetosonic, but, as of December 2012 [Burlaga and Ness, 2012b; Stone,

\footnotetext{
${ }^{1}$ Department of Earth and Space Sciences, UCLA, Los Angeles, California, USA.

${ }^{2}$ Department of Atmospheric, Oceanic and Space Sciences, University of Michigan, Ann Arbor, Michigan, USA.

Corresponding author: M. G. Kivelson, Department of Earth and Space Sciences, UCLA, Los Angeles, CA 90095, USA. (mkivelson@igpp.ucla.edu)

(C)2013. American Geophysical Union. All Rights Reserved. 2169-9380/13/10.1002/2013JA019130
}

2012], neither spacecraft had yet encountered the heliopause. Thus, the properties of the LISM are known only through remote sensing, and consequently, the properties of the external plasma of the LISM are still uncertain. Global simulation models of the heliosphere have been developed to understand the LISM interaction with the heliosphere (see, for example, the comprehensive reviews of Zank [1999] and Zank et al. [2009]), and most recent models adopt upstream conditions with the magnetosonic Mach number, $M_{m s}$, less than 1 but are less clear on the Alfvén Mach number, $M_{A}$.

[3] Although there are, as yet, no direct measurements of the properties of the heliopause or the LISM, properties of the region beyond the termination shock have been remotely sensed through measurements made deep within the heliosphere. Energetic neutral atoms (ENAs) produced by charge exchange with energetic ions in the inner heliosheath (between the termination shock and the heliopause) can travel to the center of the solar system over a hundred or more AU through the solar wind, their paths unaffected by the tenuous medium through which they move. Consequently, NASA's Interstellar Boundary Explorer (IBEX) [McComas et al., 2009a] in an elliptical orbit around the Earth and the Cassini Orbiter at Saturn [Krimigis et al., 2009] have measured the flux of ENAs formed in the outermost region of the heliosphere and/or in the LISM. Examples of the distribution of ENAs from these distant regions and the energy dependence and temporal variation of the distributions are provided by McComas et al. [2009b], Funsten et al. [2009], 

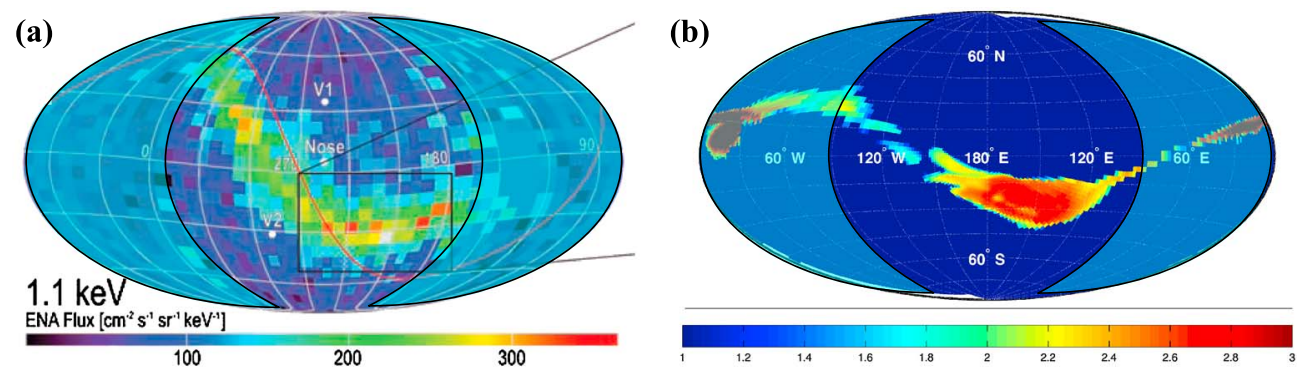

(c)

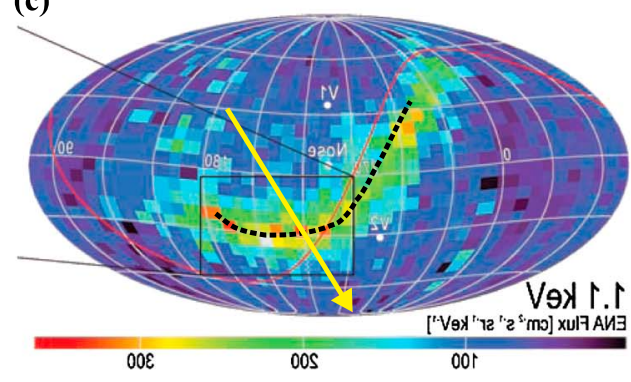

(d)

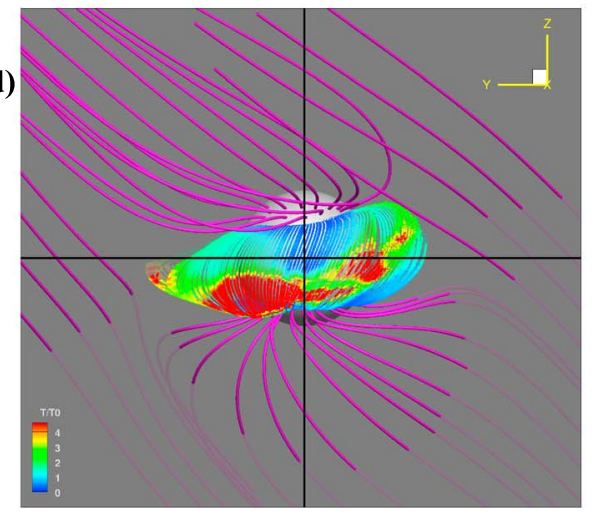

Figure 1. All-sky maps of ENA fluxes and temperature distributions. (a) Reproduced from McComas et al. [2009b, Figure 1] from whom we quote: "IBEX all-sky maps of measured ENA fluxes in Mollweide projections in ecliptic coordinates (J2000), where the heliospheric nose is near the middle and the tail extends along both sides. The pixels are $6^{\circ}$ in spin phase (latitude), with widths (longitude) determined by the spacecraft pointing for different orbits. Maps are shown in the spacecraft frame for passband central energies from IBEX-Hi." This is a map of $1.1 \mathrm{keV}$ ENAs. Labels identify the nose of the heliosphere and the directions of the trajectories of the Voyager 1 and 2 spacecraft. Color represents the flux in $\mathrm{cm}^{-2} \mathrm{~s}^{-1} \mathrm{sr}^{-1} \mathrm{keV}^{-1}$. (b) The analogous map extracted from an MHD simulation of Ganymede's magnetosphere [Jia et al., 2009] with the parameters given in the caption to Figure 2: The regions of high temperature on the magnetopause have been traced radially to the center of Ganymede assuming that ENA fluxes viewed from the interior of the magnetospheric cavity are proportional to temperature. In both images, transparent crescents are overlaid at longitude angles more than $90^{\circ}$ from the nose to remind the viewer that downstream conditions differ greatly in the two systems, implying that comparisons become less relevant with increasing distance from the nose. (c) ENA map from Figure 1a flipped to represent a view from upstream of the heliosphere, with a dashed line identifying the peak flux and a yellow arrow indicating a proposed orientation of the magnetic field of the LISM. (d) View from upstream from an MHD simulation of Ganymede's magnetosphere showing magenta magnetic field lines (shaded as they pass behind the $Y Z$ plane of the simulation), with color representing temperature relative to the background temperature. In the simulation, the field orientation is as in Figure 2c.

Fuselier et al. [2009], and Schwadron et al. [2009]. Several years of observations have been made available for study by McComas et al. [2012b].

[4] ENA emissions are particularly intense along a localized, ribbon-like region shifted away from the nose of the heliosphere as shown in the distribution of $1.1 \mathrm{keV}$ ENAs from McComas et al. [2009b] in Figure 1a (referred to as the Ribbon by McComas et al. [2012b]). The origin of the intense emissions has not been established. We suggest that localized intensification of ENA emission occurs on the heliopause where magnetic reconnection between the fields of the LISM and the heliosphere heats ions. We argue that the location and extent of the region of reconnection can be understood if the LISM flow is sub-Alfvénic. Only one other magnetosphere is embedded in a sub-Alfvénic plasma: the mini-magnetosphere of Ganymede. We use a global magnetohydrodynamic (MHD) model of Ganymede's plasma environment to establish that, for an appropriately selected orientation of the upstream field, the distribution of heated plasma on the magnetopause is similar to the distribution of ENA sources on the heliopause. We show that a superAlfvénic upstream flow would lead to a very different distribution of heated plasma on the magnetopause. Sub-Alfvénic conditions allow one to infer the orientation of the upstream magnetic field from the ENA distribution and to speculate on the large-scale form of the heliosphere.

\section{Magnetospheric Symmetry and Parameters of the Upstream Plasma}

[5] Considerable insight into the properties of any physical system is afforded by symmetry considerations, and magnetospheric structure is no exception. Quantities that affect the form of the spatial domain carved out of a flowing, external plasma surrounding a magnetized or conducting body include such properties of the external plasma as its density (number 

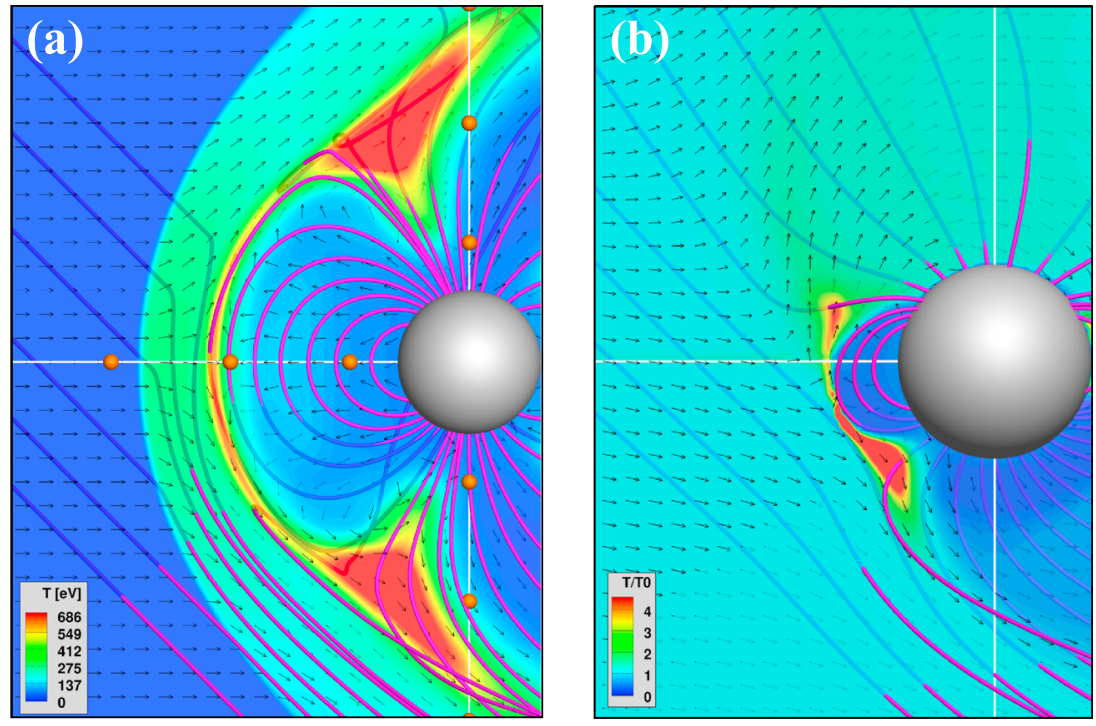

Figure 2. (a) Earth's magnetosphere in the $X Z$ plane of the GSM coordinate system (x into the flow and the dipole moment along $\mathbf{z}$ ) for a simulation run with $M_{A}=8, M_{m s}=6$. Black lines with arrows represent magnetic field lines and color represents the plasma temperature in electron volts (thermal pressure/density). Arrows are unit vectors aligned with the flow. The solar wind density, flow speed, and thermal pressure were $5 \mathrm{amu} \mathrm{cm}{ }^{-3}, 400 \mathrm{~km} \mathrm{~s}^{-1}$, and $0.007 \mathrm{nPa}$, respectively. The solar wind magnetic field components were $B_{x}=3 \mathrm{nT}, B_{y}=-3 \mathrm{nT}$ and, $B_{z}=-3 \mathrm{nT}$ in GSM coordinates. (b) Ganymede's magnetosphere for a run with $M_{A}=0.4, M_{m s}=0.3$. Magenta lines represent magnetic field lines and color again represents the plasma temperature normalized to the background value. The upstream plasma density, flow speed, and thermal pressure were $54 \mathrm{amu} / \mathrm{cm}^{-3}, 140 \mathrm{~km} \mathrm{~s}^{-1}$, and $3.8 \mathrm{nPa}$, respectively. The upstream magnetic field components were $B_{x}=60 \mathrm{nT}, B_{y}=-60 \mathrm{nT}$, and $B_{z}=-84 \mathrm{nT}$ in a right-handed coordinate system with $\mathbf{z}$ along the spin axis and $\mathbf{x}$ positive in the direction of upstream flow.

density, $n_{\text {ext }}$; mass density, $\left.\rho_{\text {ext }}\right)$ and thermal pressure $\left(p_{\text {ext }}\right)$, but it is the two vector quantities, $\mathbf{u}_{\text {ext }}$, its flow velocity relative to the central body, and $\mathbf{B}_{\text {ext }}$, its magnetic field, that are central to establishing the symmetry of the global structure of the magnetopause and constraining the locus of reconnection. Dominant aspects of the interaction of the external and internal plasmas can be characterized in terms of the dimensionless quantities: $M_{A}=\mu / \mathrm{v}_{\mathrm{A}}$, with $\mathrm{v}_{\mathrm{A}}=B /\left(\mu_{\mathrm{o}} \rho\right)^{1 / 2}$, and $\beta=p_{\text {ext }} /\left(B^{2} / 2 \mu_{\mathrm{o}}\right)$, the ratio of thermal to magnetic pressure. $M_{A}$ parameterizes the relative importance of $\mathbf{u}_{\text {ext }}\left(M_{A}>1\right)$ and $\mathbf{B}_{\text {ext }}\left(M_{A}<1\right)$ in establishing magnetospheric symmetry. The magnetosonic Mach number and $\beta$ appear to control the efficiency of magnetic reconnection between internal and external magnetic fields [Scurry and Russell, 1991; Swisdak et al., 2010; Phan et al., 2010]. The significance of $\mathbf{B}_{\text {ext }}$ in accounting for global asymmetries of the heliopause has previously been emphasized, for example, by Opher et al. [2006, 2007, 2009a], Pogorelov et al. [2008], Heerikhuisen et al. [2010], and Swisdak et al. [2010].

[6] In order to illustrate how symmetry near the upstream magnetopause depends on upstream plasma conditions, we compare properties at the magnetopause boundary using examples from simulations of magnetospheres that have been extensively studied. A useful tool for analysis of processes taking place on the external boundaries of a magnetosphere is an MHD simulation. Such simulations imperfectly represent the physical processes that act in a magnetosphere, especially such processes as magnetic reconnection and the contribution of pick-up ions that act on kinetic scales. On the other hand, MHD simulations treat global scale systems in full three dimensions and have contributed considerable insight into magnetospheric dynamics and plasma heating. Their results typically reproduce important features of spacecraft measurements and address the question of where reconnection occurs on the magnetopause for specified upstream conditions.

[7] For planetary magnetospheres (and quasi-magnetospheres such as those surrounding Mars and Venus) embedded in the super-Alfvénic $\left(M_{A}>>1\right)$ solar wind, simulations show that the thermal plasma distributions near their boundaries are close to symmetric about the flow direction regardless of the orientation of the upstream magnetic field. An example extracted from the global, 3-D MHD model BATSRUS (Block Adaptive Tree Solar wind Roe-type Upwind Scheme) of the terrestrial magnetosphere [Powell et al., 1999; Gombosi et al., 2002, 2004; Ridley et al., 2004; Tóth et al., 2012] for $M_{A}=8$ is illustrated in Figure $2 \mathrm{a}$. A cut through the noonmidnight meridian plane of the simulation shows that temperature perturbations near the dayside magnetopause are close to symmetric about the flow direction even when the external magnetic field is significantly inclined. Ganymede's magnetosphere provides an example of a magnetosphere that forms in a sub-Alfvénic $\left(M_{A}<1\right)$ flow, that of Jupiter's magnetosphere. Figure $2 \mathrm{~b}$ shows a cut through the plane of the flow and Ganymede's spin axis from a Ganymede simulation [Jia et al., 2009] with $M_{A}=0.4$ and nearly the same upstream field orientation used for the terrestrial case. Here the symmetry of the high-temperature regions is dominated by the orientation of the external magnetic field. Following convention in our coordinate system for Ganymede simulations, $\mathbf{x}$ is positive along the flow direction, $\mathbf{z}$ is along the spin axis of Ganymede, 
and $\mathbf{y}$ completes the right-handed coordinate system. For simulations of Earth's magnetosphere, $\mathbf{x}$ is negative along the flow direction, $\mathbf{z}$ is along the spin axis, and again $\mathbf{y}$ completes the right-handed coordinate system. The upstream fields are selected so that $B_{x} / B_{z}=1, B_{y} / B_{x}=-1$ for Earth and $B_{x} /$ $B_{z}=-0.7, B_{y} / B_{x}=-1$ for Ganymede. This field orientation is within a few degrees of the field orientation used by Opher et al. [2009a] and estimates of Heerikhuisen et al., [2010], Swisdak et al. [2010], and Heerikhuisen and Pogorelov [2011], all of whom provide field orientations near $40^{\circ}$ latitude, $225^{\circ}$ longitude in an ecliptic coordinate system. (The sign of the field has not been established.)

[8] Both images are cut off in the downstream region where symmetries are dictated by the very different internal field configurations. In both figures, heated plasma is found in the cusp regions. Equatorward of the cusp, heating on the dayside magnetopause is greatest just slightly southward of the nose in the case of Earth and far south of the nose for Ganymede. It is striking that the hot, upstream region in the Ganymede case falls close to the places where an unperturbed upstream field line would be tangent to the magnetopause. The simulations demonstrate that for large $M_{A}$, the symmetry of the magnetopause is largely determined by $\mathbf{u}_{\text {ext }}$, whereas for small $M_{A}$, the symmetry is largely determined by $\mathbf{B}_{\text {ext }}$.

[9] The symmetry of the most intense ENA emissions in IBEX maps [e.g., McComas et al., 2009b, 2012b] is not imposed by the flow of the LISM nor is it ordered by either the ecliptic plane or the galactic plane. However, analogy to the magnetospheric responses at Ganymede shown in Figure 1 suggests that an offset ribbon of emission could arise from reconnection of the heliospheric magnetic field with the magnetic field of a sub-Alfvénic LISM.

\section{Inferences Regarding the LISM From the Symmetry of the ENA Ribbon}

[10] In the maps of ENA emission such as that reproduced in Figure 1a, color represents intensity of the flux. The Ribbon traced out by the most intense ENA fluxes has its peak intensity localized south of the nose and shifted to the right in this view outward from the center of the heliosphere. McComas et al. [2009b] present six possible explanations for the origin of ENAs in the kiloelectron volt energy range. One suggestion is that if cold interstellar neutrals drift from the LISM into the heliosphere, they can charge exchange with solar wind ions. In a $400 \mathrm{~km} \mathrm{~s}^{-1}$ solar wind, the newly created ions (pickup ions) acquire a thermal energy close to $1 \mathrm{keV}$ and become part of the solar wind plasma, flowing outward from the Sun. Ions can be further heated as they cross the termination shock. As they approach the heliopause, a second charge exchange can create a neutral atom with energy in the kiloelectron volt range, and some of these ENAs will be heading toward the inner solar system, where their flux can be recorded by IBEX. It is this interpretation on which we build a picture that accounts for the intense source of ENAs in the localized region of the Ribbon.

[11] Although the second charge exchange described by McComas et al. [2009b] can occur anywhere beyond the termination shock, we suggest that exceptionally intense ENA flux comes from regions on the heliopause where ions are heated by magnetic reconnection. By comparing the outcomes of upstream reconnection at Earth and Ganymede, we observe that a shift of the region of reconnection from the nose of the magnetopause requires that $M_{A}$ of the LISM be less than 1 . In this situation the field of the LISM rather than the flow dominates the external dynamics and there is no upstream shock (although Zieger et al. [2013] point out that a slow mode shock of limited spatial extent can be present). If the LISM approaches the heliopause without passing through a shock, its field orientation will be little modified from its unperturbed orientation and the upstream magnetic field will first encounter the heliopause along a ribbon-like swath where it is tangent to the surface of the heliopause. In this region, the plasma could be heated by reconnection and charge exchange would then form a localized source of ENAs, which, we will argue, will fall in the kiloelectron volt energy range. The tangent surface would dictate both the center of emission and its transverse extent. Magnetic reconnection for the case of a small upstream $M_{A}$ would provide a physical explanation of the observation [McComas et al., 2012b] that ". . . the IBEX Ribbon . . . appears to be ordered by the most likely direction of the draped interstellar magnetic field".

[12] The shape of the upstream heliopause near the nose can be approximated as a spherical surface with a radius of curvature $>100 \mathrm{AU}$. If the external magnetic field is tangent to the surface in the region of the most intense ENA flux, the location of the ribbon implies that the magnetic field has an orientation roughly given by $\mathbf{B}=B_{o}(1,-1,-1)$ for an unspecified $B_{o}$ (the coordinate system here is similar to that used in Ganymede's simulation: $\mathbf{x}$ is positive along the LISM flow direction relative to the Sun's motion, $\mathbf{z}$ is along the normal of the ecliptic plane, and $\mathbf{y}$ completes the righthanded coordinate system). This assumption provides the field orientation but neither its magnitude nor its sign, although we do require that $B_{\mathrm{o}}>\left(2 \mu_{\mathrm{o}} \rho u^{2}\right)^{1 / 2}$ consistent with the inference that the upstream flow is sub-Alfvénic. (The lower bound of proposed LISM densities, $0.06 \mathrm{~cm}^{-3}$, implies $B_{\mathrm{o}}>0.4 \mathrm{nT}$ or $4 \mu \mathrm{G}$.)

\section{Comparison With a Ganymede Simulation}

[13] Sub-Alfvénic upstream conditions remain an assumption for the heliosphere but are known to characterize the plasma upstream of Ganymede. This suggests that we look to Ganymede as an example of what one might find at the heliopause if reconnection is playing an important role. At Ganymede, we are fortunate to have both in situ measurements from multiple Galileo flybys [e.g., Kivelson et al., 1996, 1998; Gurnett et al., 1996; Williams et al., 1997; Frank et al., 1997] and a global MHD model that has been validated by comparison of both plasma and field properties with the Galileo measurements [Jia et al., 2008, 2009, 2010].

[14] Details will surely differ between the heliosphere and Ganymede's magnetosphere, especially because of the markedly different field configurations that characterize the interiors of the two systems, so we focus on the regions near the nose of the heliopause. In choosing the simulation parameters, we note that Figure 4 of McComas et al. [2012a] places an upper limit on $M_{A}(0.94)$ but does not establish a minimum value. (The smallest value corresponding to the range plotted in the figure is 0.6 assuming that the external plasma is dominated by protons, but the magnetic field scale cuts off at a value well below the highest values proposed in the literature). 
In our simulation, we have set $M_{A}=0.4$. Noting that intense heating on the upstream magnetopause results from magnetic reconnection, we use temperature (obtained from an MHD simulation as the ratio of the thermal pressure to the number density) as a marker for the regions in which magnetic reconnection is occurring.

[15] Figure 1b was extracted from the Jia et al. [2009] MHD simulation used for Figure $2 \mathrm{~b}$, with an upstream field of $\mathbf{B}=B_{o}(1,-1,-1.43)$ and Mach numbers $M_{A}=0.4$, $M_{m s}=0.3$. This figure shows the distribution of temperature on the magnetopause sampled radially from the center of Ganymede (inside looking out) in order to show the expected sources of ENAs that would arise from charge exchange on the heliopause. The downstream portions of Figures $1 \mathrm{a}$ and $1 \mathrm{~b}$ are partially shaded, the focus of our discussion being the upstream hemispheres of the two systems. We find it striking that the "hot spots" in the two images are similar in location and in extent, and that the less intense portions of the ribbons bend upward in similar ways in the two plots. Figure 1c shows a view of the ENA source regions as they would appear from upstream of the heliopause, and Figure 1d shows the distribution of plasma temperature from the Ganymede simulation and schematic field lines as they would appear, also viewed from upstream of the magnetopause. The magenta field lines are ones that link to Jupiter. Field lines that start and end on Ganymede and cross the equatorial region very close to the magnetopause are also plotted.

[16] One may ask whether magnetic reconnection at Ganymede's magnetopause, which confines a stable, unidirectional field, can be compared with magnetic reconnection at the heliopause within which the sector structure of the solar wind might be expected to produce a time-varying field orientation. In addressing this question, we note that reconnection occurs over a large range of shear angles in our Ganymede simulation. For example, in Figure 1d, the heated region, which follows the curve along which the external field is tangent to magnetopause, extends over a considerable range of shear angles between internal and external fields. If we infer from the simulation that, for the low Alfvén Mach number case, reconnection is not strongly controlled by the shear angle between the fields on the two sides of the boundary, this would account for the fact that ENA fluxes do not give evidence of the rotating sector structure (D. J. McComas, personal communication, 2012) near the heliospheric equator. It would also explain how the Ribbon can extend across both the southern and northern hemispheres even though the fields have been found to have roughly opposite orientations in the inner heliosheath. (South of the heliographic equator, Voyager 2 data acquired from 2009.6 to 2010.3 show that in the inner heliosheath, the field was predominantly tangential and its polarity was nearly always positive, i.e., away from the Sun [Burlaga and Ness, 2011, but note that there is a typographical error in the label of their Figure 2, middle panel]. North of the heliographic equator, Voyager 1 data show that in the inner heliosheath the field was tangential and that its polarity turned persistently negative in mid-2010 and remained negative through day 235 of 2011 when the published data end [Burlaga and Ness, 2012a].) Neither of these orientations is antiparallel to the direction inferred for the field in the LISM, so if, as we believe, magnetic reconnection is relevant to the development of the ENA ribbon, it must occur over a large range of shear angles.
[17] Our analysis is based on an analogy and also requires us to assume that an MHD simulation provides a reasonably close approximation to the physics of the system being investigated. One must be cautious in doing so, because it is well known that processes near and below the scale of ion gyroradii that play important roles in reconnection are absent in an MHD model. Processes below the MHD scale are crudely represented in our simulation by incorporating a current-dependent anomalous resistivity model, which is switched on in regions with strong localized currents [Jia et al., 2009]. It is possible that such an MHD model may not provide an accurate representation of the regions in which reconnection occurs. However, we can argue that the fact that reconnection is present in regions where there is considerable shear between the internal and external fields is not unique to MHD simulations. Kinetic simulations [Swisdak et al., 2010] and observations in space plasmas [Phan et al., 2010] have revealed that reconnection occurs for a large range of shear angles between the magnetic fields on the two sides of the reconnection line provided that the plasma $\beta$ does not differ too greatly on the two sides. We can estimate the $\beta$ on the two sides of the heliopause, although the parameters are uncertain. In the inner heliosheath, using plasma parameters from Richardson [2012] (density $\sim 0.002 \mathrm{~cm}^{-3}$, temperature $6 \times 10^{4} \mathrm{~K}$ ) and magnetic field magnitude from Burlaga and Ness [2012a] $(B \sim 0.2 \mathrm{nT})$, we estimate that $\beta \sim 0.1$. In the LISM, using the parameters recently adopted by Zank et al. [2013] (his Model 3), we find $\beta \sim 0.065$. Figure 3 of Phan et al. [2010] shows that for a change of $\beta$ of 1 or less, reconnection is observed for all shear angles above a cutoff near $50^{\circ}$, and for changes of the order of 0.1 , there are effectively no constraints on the shear angle. Thus, it is likely that reconnection can occur where the upstream flow first encounters the boundary for either toward or away orientation of the field within the heliopause. If our model of the Ribbon is correct, reconnection occurs on the heliopause near the tangent curve defined geometrically by the external field and has little additional dependence on the angle between the external and internal fields. The rate of reconnection may be controlled by the shear angle between the reconnecting fields and by various plasma parameters. The plasma parameters near the heliopause are uncertain, with the uncertainty increased by the unknown contribution of pickup ions near the heliopause. It, therefore, follows that, if our model proves to be correct, the arguments of Swisdak et al. [2010] and Phan et al. [2010] may help constrain such plasma parameters as $\beta$ and the change of $\beta$ across the boundary, requiring them to lie in a range that permits reconnection over a large range of shear angles of the reconnecting fields.

\section{Reconnection and the Increase of the Width of the ENA Ribbon With Energy}

[18] A feature of the ENA ribbon that we have not yet discussed is its energy dependence. McComas et al. [2012b] combined data from 3 years of measurements to obtain maps of the emissions at different energies between $0.7 \mathrm{keV}$ and $4.3 \mathrm{keV}$. A set of those maps is reproduced in Figure 3. Focusing on the upstream hemisphere, we have superimposed curves to indicate where we propose that reconnection would be centered. Within the upstream portion of the images, the same curve provides a reasonably good fit to the spatial 


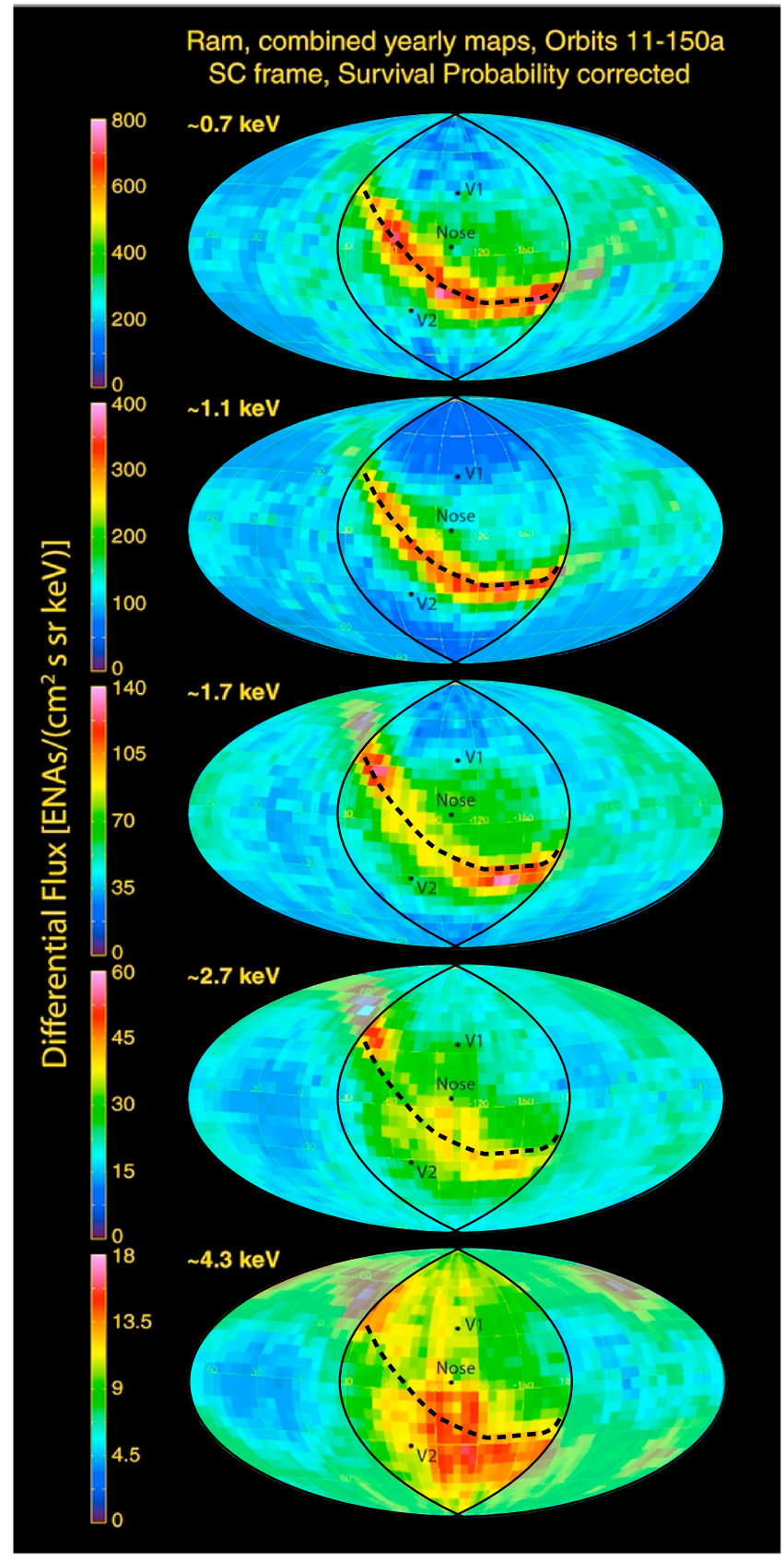

Figure 3. Maps (inside looking out) of ENA fluxes at energies from $0.7 \mathrm{keV}$ to $4.3 \mathrm{keV}$ obtained by combining annual maps over 3 years reproduced from McComas et al. [2012b, Figure 17]. A dashed black curve traces the center of emissions at $0.7 \mathrm{keV}$ and has been superimposed on the higher energy plots. As in Figure 1, the downstream regions more than $90^{\circ}$ from the meridian close to the nose are masked.

distribution of the peak fluxes at all energies, as would be expected if the anomalies relate to reconnection. At energies above $1.1 \mathrm{keV}$, the spread about the curve of the region of intense flux increases with energy.

[19] Let us consider how reconnection can account for the ENA flux maps of Figure 3. First, one must ask why reconnection leads to an increase of ENA flux. In answering this question, we first assume that the phase space distribution of ions in the kiloelectron volt range decreases with increasing energy. If so, processes that increase ion energies result in an increase of phase space density at fixed energy. Reconnection is such a process. Ions moving along a reconnected magnetic field from outside to inside the heliopause (or vice versa) are reflected from the kink in the field and emerge with their parallel velocity increased of the order twice the Alfvén speed on the slower side of the interface. (This is consistent with Cowley's [1982] comment that ion energy gain produced by subsolar reconnection is expected to be of the order of the field energy.) If, as we propose, the upstream Alfvén Mach number is of order 1, the parallel energy of a typical ion would increase in the reconnection process by the order of

$$
\Delta E=\frac{m}{2}\left[\left(v_{\text {in }}+\Delta v\right)^{2}-v_{\text {in }}^{2}\right]=E_{0}\left(\frac{2 \Delta v}{v_{\text {in }}}\right)=E_{0}\left(\frac{4 v_{A}}{v_{\text {in }}}\right)=4 E_{0} / M_{A}
$$

implying that ion energy roughly quadruples as a result of reconnection. Near the site of reconnection, the number of particles of kiloelectron volt range energy increases relative to the number in the background distribution.

[20] Reconnection initially increases the parallel velocity of ions, meaning that newly accelerated ions flow along the heliopause with no significant perpendicular energy. In order to produce ENAs moving inward toward IBEX, ions must acquire significant perpendicular energy. Pitch angle scattering is, therefore, required. Kinetic simulations [Lottermoser and Scholer, 1998] show that ions moving outward from a reconnection line are isotropized by nonlinear effects. Already at 200 ion inertial lengths or less from the neutral line, the ion pitch angle distribution has become quasi-isotropic. At the heliopause, the ion inertial length is of order $1000 \mathrm{~km}$ or $10^{-5}$ AU. This implies that change exchange can produce ENAs moving both along and radially away from the heliopause very close to the reconnection site.

[21] It remains to be argued why the intensity and the width of the ribbon vary with energy. Using the charge exchange cross sections of Lindsay and Stebbings [2005], we estimate that in the range $1-10 \mathrm{keV}$, the cross section decreases with energy as $\sim E^{-0.3}$, i.e., a factor of 2 over the full range or a factor of 0.66 for a factor of 4 increase of energy. A decrease of cross section combined with a decrease of the unperturbed ion population with energy is likely to account for the rapid decrease of the intensity of peak ENA flux with energy in Figure 3, where the color scales differ for the different maps.

[22] The decrease of the charge exchange cross section with energy can also account for the energy-dependent latitudinal spread of ENA flux. As the cross section decreases, the distance that an ion travels before converting into a neutral increases. Thus, if the acceleration occurs near the reconnection point (dashed contours in Figure 3), the width of the region of significant charge exchange becomes increasingly broad about those contours as the ion energy increases and the charge exchange cross section decreases.

\section{Speculations on the Shape of the Global Heliosphere}

[23] Our suggestion that the flow of the LISM is subAlfvénic has an additional implication. The shape of the magnetopause of Ganymede differs considerably from the 


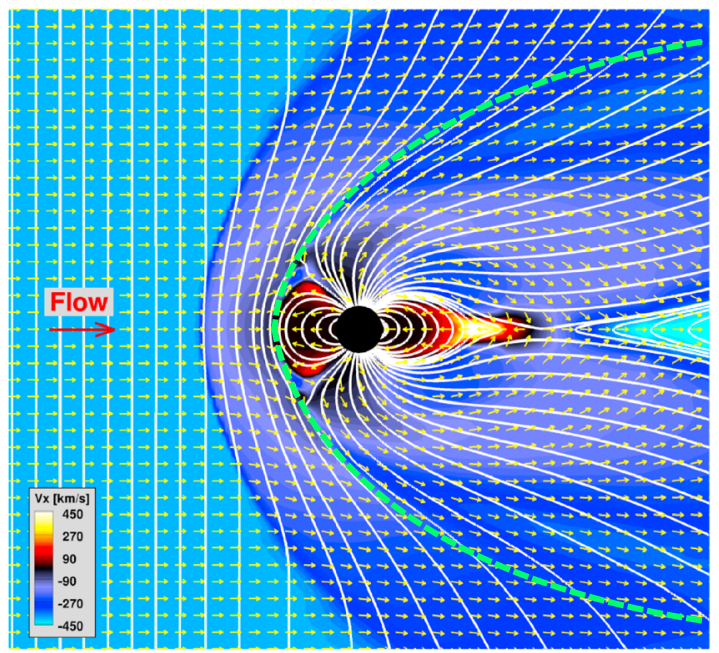

(a)

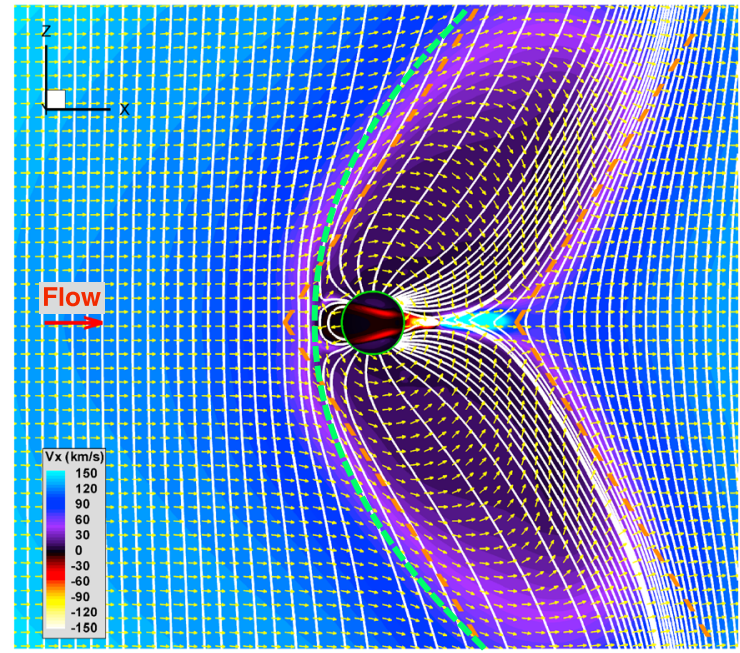

(b)

Figure 4. MHD simulations of magnetospheres formed in flows of different Alfvén Mach numbers. (a) A simulation of Earth's magnetosphere for $M_{A}=2$. (b) A simulation of Ganymede's magnetosphere for $M_{A}=0.4$. In both cases, white lines represent the magnetic field, which is southward oriented in the incident flow, and color represents flow speeds. Yellow unit vectors are aligned with the flow. The dashed green lines delineate the magnetopause.

bullet shape of a magnetosphere embedded in a super-Alfvénic flow, simply because, on any flux tube, the interface between the internal and external fields moves outward along the field at the speed of an Alfvén wave, while the flux tube convects tailward at the speed of the external flow. For Earth, the flow speed is much larger than the wave speed, so the interface convects tailward too rapidly for the magnetopause to flare substantially. At Ganymede, the wave speed is faster than the flow speed, so the interface moves faster in the $z$ direction than in the direction of the flow, producing a highly flared interface. Examples of magnetospheres formed for different values of $M_{A}$ are shown in Figure 4. In Figure 4a, the Earth's magnetosphere is modeled for the low, but still super-Alfvénic, value of $M_{A}=2$. The downstream boundary is highly confined in the $z$ direction. In Figure 4b, Ganymede's magnetosphere is simulated for $M_{A}=0.4$. The magnetopause flares at a large angle from the external flow direction. As we propose a relatively low $M_{A}$ for the LISM, we anticipate that for the heliosphere, the boundary will have characteristics similar to that of Figure $4 \mathrm{~b}$, with the asymptotic angle between the flow direction and the heliopause possibly larger than $45^{\circ}$, purely because of the rapid propagation of Alfvénic disturbances relative to the flow speed. The tilt of the field, coupled with the relatively rapid propagation of the reconnected portion of a flux tube, will enhance the north-south asymmetries of the system as has been illustrated in the work of Opher et al. [2009b]. Another notable difference between $M_{A}=2$ and 0.4 is the shape of the upstream boundary, which is distinctly rounded in Figure $4 \mathrm{a}$ but far more blunt in Figure 4b. This is consistent with recent comments presented in connection with a discussion of the absence of a bow shock upstream of the heliopause [McComas et al., 2012a].

\section{Discussion}

[24] The Galileo Orbiter made multiple close passes by Ganymede, collecting data that revealed many features of
Ganymede's plasma and field environment, characterizing what, to this time, is a unique example of a magnetosphere embedded in a sub-Alfvénic flow. Simulations of the system that successfully reproduce critical aspects of the measurements give insight into the structure and dynamics of the magnetosphere. Drawing upon Ganymede studies, we have put forward an interpretation of aspects of the interaction of the heliosphere with the flowing plasma of the LISM. Our principal conclusions are that the upstream flow is subAlfvénic, that the orientation of the field in the LISM can be inferred from the distribution of the peak ENA fluxes to within a sign, and that magnetic reconnection is a likely mechanism for the generation of the ENAs. We also predict that the heliopause flares far more than does a typical planetary magnetopause. We eagerly await direct measurements of the properties of the LISM as the Voyager spacecraft continue their journey away from the Sun.

[25] Acknowledgments. The coauthors of this paper are deeply grateful to Dave McComas for encouraging us to put forward the ideas of this paper, and to him and the IBEX team for making their data comprehensible and widely available. Our work rests heavily on simulation tools developed by Tamas Gombosi and colleagues and we are grateful to them for their support and their generosity.

[26] Masaki Fujimoto thanks Merav Opher and an anonymous reviewer for their assistance in evaluating this paper.

\section{References}

Burlaga, L. F., and N. F. Ness (2011), Transition from the sector zone to the unipolar zone in the heliosheath: Voyager 2 magnetic field observations, Astrophys. J., 737, 35, doi:10.1088/0004-637X/737/1/35

Burlaga, L. F., and N. F. Ness (2012a), Heliosheath magnetic fields between 104 and $113 \mathrm{AU}$ in a region of declining speeds and a stagnation region, Astrophys. J., 749, 13, doi:10.1088/0004-637X/749/1/13

Burlaga, L. F., and N. F. Ness (2012b), Heliosheath magnetic fields between $\sim 105$ and 120 AU, Abstract SH14B-01, Presented at Fall Meeting, AGU, San Francisco, December 2012.

Cowley, S. W. H. (1982), The causes of convection in the Earth's magnetosphere: A review of developments during the IMS, Rev. Geophys. Space Phys., 20, 531-565, doi:10.1029/RG020i003p00531. 


\section{KIVELSON AND JIA: THE SMALLEST AND LARGEST MAGNETOSPHERES}

Frank, L. A., W. R. Paterson, K. L. Ackerson, and S. J. Bolton (1997), Outflow of hydrogen ions from Ganymede, Geophys. Res. Lett., 24(17), 2151-2154.

Funsten, H. O., et al. (2009), Structures and spectral variations of the outer heliosphere in IBEX energetic neutral atom maps, Science, 326, 964.

Fuselier, S. A., et al. (2009), Width and variation of the ENA flux ribbon observed by the Interstellar Boundary Explorer, Science, 326, 962, doi:10.1126/science.1180981.

Gombosi, T. I., G. Tóth, D. L. De Zeeuw, K. C. Hansen, K. Kabin, and K. G. Powell (2002), Semi-relativistic magnetohydrodynamics and physics-based convergence acceleration, J. Comput. Phys., 177, 176, doi:10.1006/jcph.2002.7009.

Gombosi, T. I., et al. (2004), Solution-adaptive magnetohydrodynamics for space plasmas: Sun-to-Earth simulations, Comput. Sci. Eng., 6(2), 14 doi:10.1109/MCISE.2004.1267603

Gurnett, D. A., W. S. Kurth, A. Roux, S. J. Bolton, and C. F. Kennel (1996) Evidence for a magnetosphere at Ganymede from plasma-wave observations by the Galileo spacecraft, Nature, 384, 535-537.

Heerikhuisen, J., and N. V. Pogorelov (2011), An estimate of the nearby interstellar magnetic field using neutral atoms, Astrophys. J., 738, 29, doi:10.1088/0004-637X/738/1/29.

Heerikhuisen, J., N. V. Pogorelov, G. P. Zank, G. B. Crew, P. C. Frisch H. O. Funsten, P. H. Janzen, D. J. McComas, D. B. Reisenfeld, and N. A. Schwadro (2010), Pick-up ions in the outer heliosheath: A possible mechanism for the Interstellar Boundary Explorer ribbon, Astrophys. J., 708, L126-L130, doi:10.1088/2041-8205/708/2/L126.

Jia, X., R. J. Walker, M. G. Kivelson, K. K. Khurana, and J. A. Linker (2008), Three-dimensional MHD simulations of Ganymede's magnetosphere, J. Geophys. Res., 113, A06212, doi:10.1029/2007JA012748.

Jia, X., R. J. Walker, M. G. Kivelson, K. K. Khurana, and J. A. Linker (2009), Properties of Ganymede's magnetosphere inferred from improved three-dimensional MHD simulations, J. Geophys. Res., 114, A09209, doi:10.1029/2009JA014375.

Jia, X., R. J. Walker, M. G. Kivelson, K. K. Khurana, and J. A. Linker (2010), Dynamics of Ganymede's magnetopause: Intermittent reconnection under steady external conditions, J. Geophys. Res., 115, A12202, doi:10.1029/ 2010JA015771.

Kivelson, M. G., K. K. Khurana, C. T. Russell, R. J. Walker, J. Warnecke, F. V. Coroniti, C. Polanskey, D. J. Southwood, and G. Schubert (1996), Discovery of Ganymede's magnetic field by the Galileo spacecraft Nature, 384, 537.

Kivelson, M. G., J. Warnecke, L. Bennett, S. Joy, K. K. Khurana, J. A. Linker, C. T. Russell, R. J. Walker, and C. Polanskey (1998), Ganymede's magnetosphere: Magnetometer overview, J. Geophys. Res., 103(E9), 19,963.

Krimigis, S. M., D. G. Mitchell, E. C. Roelof, K. C. Hsieh, and D. J. McComas (2009), Imaging the interaction of the heliosphere with the interstellar medium from Saturn with Cassini, Science, 326, 971, doi: $10.1126 /$ science. 1181079

Lindsay, B. G., and R. F. Stebbings (2005), Charge transfer cross sections for energetic neutral atom data analysis, J. Geophys. Res., 110, A12213, doi:10.1029/2005JA011298

Lottermoser, R.- F., and M. Scholer (1998), Ion kinetic effects in magnetic reconnection: Hybrid simulations, J. Geophys. Res., 103(A3), 4547-4559.

McComas, D. J., et al. (2009a), IBEX: Interstellar Boundary Explorer, Space Sci. Rev., 146, 11, doi:10.1007/s11214-009-9499-4.

McComas, D. J., et al. (2009b), Global observations of the interstellar interaction from the interstellar boundary explorer (IBEX), Science, 326, 959 , doi:10.1126/science. 1180906 .
McComas, D. J., et al. (2012a), The heliosphere's interstellar interaction: No bow shock, Science, 336, 1291, doi:10.1126/science.1221054.

McComas, D. J., et al. (2012b), The first three years of IBEX observations and our evolving heliosphere, Astrophys. J. Suppl., 203, 1, doi:10.1088/ 0067-0049/203/1/1

Opher, M., E. C. Stone, and P. C. Liewer (2006), The Effects of a Local Interstellar Magnetic Field on Voyager 1 and 2 Observations, Astrophys. J. Lett, 640, L71-L74.

Opher, M., E. C. Stone, and T. Gombosi (2007), The orientation of the local interstellar magnetic field, Science, 316, 875, doi:10.1126/science.1139480.

Opher, M., J. D. Richardson, G. Tóth, and T. I. Gombosi (2009a) Confronting observations and modeling: The role of the interstellar magnetic field in Voyager 1 and 2 asymmetries, Space Sci. Rev., 143, 43.

Opher, M., F. Alouani Bibi, G. Toth, J. D. Richardson, V. V. Izmodenov, and T. I. Gombosi (2009b), A strong, highly-tilted interstellar magnetic field near the solar system, Nature, 462, doi:10.1038/nature08567.

Phan, T.-D., J. T. Gosling, G. Paschmann, C. Pasma, J. F. Drake, M. Øieroset D. Larson, R. P. Lin, and M. S. Davis (2010), The dependence of magnetic reconnection on plasma $\beta$ and magnetic shear: Evidence from solar wind observations, Astrophys. J. Lett., 719, L199-L203, doi:10.1088/2041-8205/ 719/2/L199.

Pogorelov, N. V., J. Heerikhuisen, and G. P. Zank (2008), Probing heliospheric asymmetries with an MHD-Kinetic model, Astrophys. J., 675, L41-L44.

Powell, K. G., P. L. Roe, T. J. Linde, T. I. Gombosi, and D. L. DeZeeuw (1999)

A solution-adaptive upwind scheme for ideal magnetohydrodynamics, J. Comput. Phys., 154, 284-309.

Richardson, J. D. (2012), Voyager observations of the interaction of the heliosphere with the interstellar medium, J. Adv. Res., 4, 229-233, doi:10.1016/j.jare.2012.09.002.

Ridley, A., T. Gombosi, and D. Dezeeuw (2004), Ionospheric control of the magnetosphere: Conductance, Ann. Geophys., 22, 567-584.

Schwadron, N. A., et al. (2009), Comparison of Interstellar Boundary Explorer observations with 3D global heliospheric models, Science, 326 , 966, doi:10.1126/science.1180986.

Scurry, L., and C. T. Russell (1991), Proxy studies of energy transfer to the magnetosphere, J. Geophys. Res., 96(A6), 9541, doi:10.1029/91JA00569.

Stone, E. (2012), Voyager 1 observations of rapid changes in the heliosheath, Abstract SH13D-01, Presented at Fall Meeting, AGU, San Francisco, December 2012

Swisdak, M., M. Opher, J. F. Drake, and F. Alouani Bibi (2010), The vector direction of the interstellar magnetic field outside the heliosphere Astrophys. J., 710, 1769, doi:10.1088/0004-637X/710/2/1769.

Tóth, G., et al. (2012), Adaptive numerical algorithms in space weather modeling, J. Comput. Phys., 231, 870, doi:10.1016/j.jcp.2011.02.006.

Williams, D. J., et al. (1997), Energetic particle signatures at Ganymede Implications for Ganymede's magnetic field, Geophys. Res. Lett., 24(17), 2163-2166.

Zank, G. P. (1999), Interaction of the solar wind with the local interstellar medium: A theoretical perspective, Space Sci. Rev., 89, 413.

Zank, G. P., N. V. Pogorelov, J. Heerikhuisen, H. Washimi, V. Florinski, S. Borovikov, I. Kryukov, and H. R. Müller (2009), Physics of the solar wind-local interstellar medium interaction: Role of magnetic fields, Space Sci. Rev., 146, 295-327, doi:10.1007/s11214-009-9497-6.

Zank, G. P., J. Heerikhuisen, B. E. Wood, N. V. Pogorelov, E. Zirnstein, and D. J. McComas (2013), Heliospheric structure: The bow wave and the hydrogen wall, Astophys. J., 763, 20, doi:10.1088/0004-637X/763/1/20.

Zieger, B., M. Opher, N. A. Schwadron, D. J. McComas, and G. Tóth (2013), A slow bow shock ahead of the heliosphere, Geophys. Res. Lett. 40, 2923-2928, doi:10.1002/grl.50576. 